\title{
Trabajo de Fin de Grado a Debate entre los profesores del Área de Ciencias Sociales y Jurídicas de la Universidad de Sevilla
}

\author{
The Debate on Dissertations among the professors of Social and \\ Legal Sciences in the University of Seville
}

\author{
José Antonio Donoso Anes (jadonoso@us.es) \\ Francisco Serrano Domínguez (fserrano@us.es) \\ José Antonio Camúñez Ruiz (camunez@us.es) \\ Universidad de Sevilla (España)
}

http://dx.doi.org/10.12795/EDUCADE.2016.i07.04

RESUMEN: Con la última ordenación académica de las enseñanzas universitarias oficiales (Real Decreto 1393/2007, de 29 de octubre) tiene su aparición la asignatura de Trabajo Fin de Grado (TFG); a través de ella se pretende que el alumno cierre su etapa de grado mediante un trabajo en el que se integren los conocimientos y las competencias adquiridas.

El objetivo del trabajo es trasladar el resultado del debate que actualmente se está teniendo en los centros de economía y derecho de la Universidad de Sevilla sobre el desarrollo de la materia de Trabajo Fin de Grado, que abarca aspectos como el desarrollo normativo, reconocimiento de créditos, asignación de trabajos, metodología de trabajo y evaluación.

El método de recogida de datos ha sido, mediante reuniones con profesores implicados en la asignatura y las diferentes comisiones de seguimiento de los TFG de los centros con los que se ha trabajado.

El resultado obtenido muestra un alto grado de confusión e insatisfacción por parte del profesorado implicado en el estudio; se propone la necesidad de unificar la normativa, la mejora en el reconocimiento, se cuestiona la obligatoriedad de su realización y de su evaluación mediante defensa oral pública.

PALABRAS CLAVE: Trabajo fin de grado, diseño curricular, trabajo fin de carrera

ABSTRACT: The last official university teaching academic plan (Royal Decree 1393/2007, of 29th. October) has meant the emergence of a Final Year Project (FYP) as a subject. Its aim is for students to finish their degree period via work which brings together the knowledge and skills which they have acquired.

This article's goal is to shift the result of the debate that is currently taking place in the centres of economics and law of the University of Seville about working out the FYP. This subject encompasses aspects such as regulatory development, credit recognition, work allocations, work methodology and evaluation.

The data collection method has been via meeting with the professors involved in the subject and the different follow-up commissions of the FYP in the centres concerned.

The result shows the high degree of confusion and dissatisfaction of the teachers involved in the study. The need to unify the regulation and to improve the recognition is proposed. The subject's mandatory nature and its evaluation via public presentation are also questioned.

Keywords: Final Year Project, curricular design, FYP debate 


\section{INTRODUCCIÓN}

Uno de los efectos de la integración de España en el Espacio Europeo de Educación Superior (EEES) ha sido la reestructuración de las titulaciones. Con ese fin se promulgó Real Decreto 1393/2007, de 29 de octubre, por el que se establece la ordenación de las enseñanzas universitarias oficiales. Entre las muchas novedades que introdujo la norma vamos a destacar la obligatoriedad de realizar un Trabajo de Fin de Grado (TFG); como parte de un sistema de aseguramiento de la calidad dentro de la nueva era de la Educación Superior (Mateo et al, 2012, p.28).

Entendemos que las universidades, los centros y los departamentos son los principales afectados por esta normativa y los responsables de una adecuada gestión de las nuevas materias que se han incorporado en los Planes de Estudio.

El objetivo del trabajo es presentar los resultados del debate que actualmente se está produciendo en nuestros centros (Facultad de Ciencias Económicas y Empresariales, Facultad de Turismo y Finanzas, Facultad de Derecho y Facultad de Ciencias del Trabajo) de la Universidad de Sevilla después de tres años de implantación. El debate alcanza a todos los niveles de la estructura universitaria: universidad, centros, departamentos, profesores y alumnos; y su contenido abarca desde los aspectos puramente normativos (reconocimiento de créditos, papel del tutor, objeto del TFG y evaluación) hasta la aportación que esta materia está realizando en la formación del alumno (trabajos monodisciplinares o multidisciplinares; trabajos de investigación o de formación hacia la preparación profesional o la inserción laboral).

La metodología de trabajo ha consistido en un análisis de la normativa (estatal e interna de universidad y de centros) que afecta a los trabajos de fin de grado; así como reuniones y entrevistas no estructuradas y dirigidas a conocer la opinión de los tutores de trabajos y/o participantes en comisiones evaluadoras y de seguimiento de los TFG.

Como principales conclusiones resaltar el profundo descontento que actualmente existe en torno a los TFG entre los profesores participantes (profesores-tutores y profesores-evaluadores) en parte por la incertidumbre derivada de la normativa reguladora, en parte por la amplia discrecionalidad que tienen tanto los tutores como evaluadores en el momento de asumir su rol respecto a la materia de TFG.

\section{NORMATIVAS REGULADORAS DE LOS TFG}

\subsection{Antecedentes}

Los trabajos de fin de carrera no son novedosos. En España, su regulación se remonta al año 1957 cuando de promulgó la Ley de 20 de julio de Ordenación de las Enseñanzas Técnicas, donde en su artículo 11.2 indicaba que "como complemento del último curso cada alumno deberá efectuar un proyecto de fin de carrera en el que acredite la formación adquirida. Su aprobación será precisa para la colación del Grado de Arquitecto o Ingeniero".

Desde entonces, teniendo en cuenta las distintas reformas del sistema educativo (Ministerio de Educación, Cultura y Deporte, 2004), el Trabajo de Fin de Carrera ha formado parte de cada uno de los planes de estudio las carreras técnicas. Su objeto era la realización de un trabajo original en el que el alumno ponía a prueba todos sus conocimientos, encaminado a la demostración de haber recibido las competencias, habilidades y habilitando a quien lo supera para el ejercicio profesional. Este referente 
ha resultado muy útil para la inclusión de los TFG en ese tipo de grados (Valderrama et al, 2009, p. 406).

Para titulaciones no técnicas existía también una figura similar al trabajo de fin de carrera, denominada "memoria de licenciatura" cuya realización no resultaba obligatoria para poder ejercer la profesión sino que se establecía como un requisito para poder acceder a los estudios de tercer ciclo.

\subsection{Normativa estatal}

El Real Decreto 1393/2007, de 29 de octubre, por el que se establece la ordenación de las enseñanzas universitarias oficiales de Grado, Master Universitario y Doctorado, modificado por el Real Decreto 861/2010, de 2 de julio, contempla la estructura a la que deben adaptarse los planes de estudios conducentes a la obtención de títulos de Grado, e indica, en el artículo 12 que las enseñanzas oficiales de Grado concluirán con la elaboración y defensa de un Trabajo de Fin de Grado (TFG) que tendrá entre 6 y 30 créditos, que deberá realizarse en la fase final del plan de estudios y estar orientado a la evaluación de competencias asociadas al título.

Coincidimos con Rekalde (2011, p. 181) que el Real Decreto no establece ningún principio orientador de cómo deben estructurarse los TFG, ni la manera de ser tutelado ni evaluado; por lo que, en buena lógica estos trabajos deben tener su referente en los ya consolidados de las grados técnicos o bien inspirados en lo realizado en otras Universidades de referencia en el entorno del EEES.

\subsection{Normativa de la Universidad de Sevilla}

La Universidad de Sevilla (US), como responsable del desarrollo de esta norma, promovió, mediante acuerdo de Consejo de Gobierno de 21 de diciembre de 2009 la normativa relativa a los trabajos de fin de grado.

En su artículo 1, relativo a su objeto, la normativa establece que "el trabajo fin de carrera consistirá en la realización por parte del estudiante, bajo la dirección de un tutor, de un proyecto, memoria o estudio sobre un tema de trabajo que se le asignará y en el que desarrollará y aplicará conocimientos, capacidades y competencias adquiridos en la titulación". Dado que esta normativa afecta a todas las facultades de la Universidad, la redacción es genérica para poder dar cabida a todas las posibilidades que puedan darse en las mismas. Por ello, cubre los ya tradicionales proyectos de fin de carrera de las titulaciones técnicas, mientras que las memorias y estudios están más bien orientados a titulaciones donde el desarrollo de un proyecto resulte más complejo (por ejemplo, en los grados asociados a las humanidades). Si nos situamos en el terreno de las Ciencias Sociales y Jurídicas, tienen cabida los tres tipos de trabajos.

Otros aspectos que quedan regulados son:

- Número de autores que puede tener un trabajo (normalmente uno; máximo tres).

- Las funciones del tutor (profesor con plena capacidad docente con una labor orientadora, supervisora y que vele por el cumplimiento de los objetivos fijados).

- Las comisiones evaluadoras y tribunales de apelación. En este sentido, la legislación de la US es más restrictiva que la nacional, por cuanto que esta establece la obligatoriedad de una defensa pública en los Trabajos de Fin de 
Máster y no para los TFG'. Así la US le ha dado carácter de defensa pública al TFG lo que impide poder utilizar sistemas alternativos para su evaluación.

- Aspectos a evaluar de los trabajos y calificación de los mismos.

- Las normativas internas de los distintos centros.

Serán las normativas internas de cada centro las que desarrollen de una manera más específica estos puntos que son abordados por la normativa general universitaria.

\subsection{Normativas internas de los centros de la US}

La normativa universitaria, en un intento de que cada titulación se adapte de la mejor manera a las exigencias de los TFG, otorga libertad para organizar esta asignatura; de tal forma que cada centro tendrá libertad para establecer:

- Los períodos de matrícula en cada curso académico, de modo que el alumno tenga derecho a tres actos de presentación por curso académico.

- Los procedimientos para la asignación y modificación, en su caso, del tutor.

- La asignación del tema del trabajo al alumnado.

- Los requisitos y plazos para el depósito del trabajo.

- La regulación del acto de presentación.

- El sistema de evaluación asociado a esa asignatura.

- La composición de la comisión evaluadora.

- Los criterios de designación de la comisión evaluadora y del tribunal de apelación.

- El procedimiento del recurso de apelación.

Tomando como referencia los centros implicados en el estudio: Facultad de Turismo y Finanzas (FTF), Facultad de Ciencias Económicas y Empresariales (FCEYE), Facultad de Derecho (FD) y Facultad de Ciencias del Trabajo (FCT), lo realmente destacable son las diferencias significativas que existen entre las normativas que cada centro se ha dotado. Hay que tener en cuenta que en la mayor parte de la docencia impartida en estas titulaciones son responsabilidad de los mismos departamentos. En consecuencia, es frecuente que docentes que imparten docencia en centros diferentes tengan que asumir TFG sujetos a normativas distintas (tabla 1).

\footnotetext{
1 El art. 12.3 del RD. 1393/2007, referente a los títulos de grado indica que "estas enseñanzas concluirán con la elaboración y defensa de un trabajo de fin de Grado" mientras de que el art. 15.3 de la misma norma, relativa a las enseñanzas universitarias oficiales de master establece que "estas enseñanzas concluirán con las elaboración y defensa pública de un trabajo de fin de Master" por lo que parece que el requisito de defensa pública de los TFG es uno más, pero no necesariamente el único.
} 
J. Antonio Donoso, Francisco Serrano, J. Antonio Camúñez

Trabajo de Fin de Grado a Debate entre los profesores del Área de Ciencias Sociales y Jurídicas de la Universidad de Sevilla

Tabla 1. Comparativa de la regulación por centros

\begin{tabular}{|c|c|c|c|c|}
\hline & FTF & FCEYE & FCT & FD \\
\hline Plazos Matrícula & $\begin{array}{l}\text { El mismo que } \\
\text { cualquier otra } \\
\text { asignatura }\end{array}$ & $\begin{array}{l}\text { El mismo que } \\
\text { cualquier otra } \\
\text { asignatura }\end{array}$ & $\begin{array}{l}\text { El mismo que } \\
\text { cualquier otra } \\
\text { asignatura }\end{array}$ & $\begin{array}{l}\text { El mismo que } \\
\text { cualquier otra } \\
\text { asignatura }\end{array}$ \\
\hline $\begin{array}{l}\text { Asignación } \\
\text { número de } \\
\text { trabajos }\end{array}$ & $\begin{array}{l}\text { La Facultad, } \\
\text { reparte entre los } \\
\text { distintos } \\
\text { Departamentos } \\
\text { en función de su } \\
\text { peso relativo en } \\
\text { la titulación }\end{array}$ & $\begin{array}{l}\text { La Facultad, } \\
\text { reparte entre los } \\
\text { distintos } \\
\text { Departamentos } \\
\text { en función de su } \\
\text { peso relativo en } \\
\text { la titulación }\end{array}$ & $\begin{array}{l}\text { La Facultad, } \\
\text { reparte entre los } \\
\text { distintos } \\
\text { Departamentos } \\
\text { en función de su } \\
\text { peso relativo en } \\
\text { la titulación }\end{array}$ & $\begin{array}{l}\text { La Facultad, } \\
\text { reparte entre los } \\
\text { distintos } \\
\text { Departamentos } \\
\text { en función de su } \\
\text { peso relativo en } \\
\text { la titulación }\end{array}$ \\
\hline Asignación Tutor & $\begin{array}{l}\text { Salvo acuerdo, el } \\
\text { alumnado elige } \\
\text { por orden de } \\
\text { expediente } \\
\text { académico. }\end{array}$ & $\begin{array}{l}\text { El alumnado } \\
\text { elige } \\
\text { Departamento } \\
\text { por orden de } \\
\text { expediente y } \\
\text { una comisión } \\
\text { interna del } \\
\text { Departamento } \\
\text { (figura creada } \\
\text { por la normativa } \\
\text { de la Facultad) } \\
\text { asigna el Tutor. }\end{array}$ & $\begin{array}{l}\text { El alumnado elige } \\
\text { Departamento } \\
\text { por orden de } \\
\text { expediente y una } \\
\text { comisión interna } \\
\text { del } \\
\text { Departamento } \\
\text { (figura creada } \\
\text { por la normativa } \\
\text { de la Facultad) } \\
\text { asigna el Tutor. }\end{array}$ & $\begin{array}{l}\text { Por acuerdo } \\
\text { entre los } \\
\text { docentes con } \\
\text { carga de TFG } \\
\text { coordinados por } \\
\text { el Decanato. }\end{array}$ \\
\hline Asignación Temas & $\begin{array}{l}\text { Salvo acuerdo } \\
\text { previo, la } \\
\text { Facultad sobre } \\
\text { propuestas de los } \\
\text { profesores, } \\
\text { remitida por la } \\
\text { dirección del } \\
\text { Departamento. }\end{array}$ & $\begin{array}{l}\text { Acuerdo Tutor- } \\
\text { alumno y en } \\
\text { ausencia de } \\
\text { éste, el tutor. }\end{array}$ & $\begin{array}{l}\text { Acuerdo Tutor- } \\
\text { alumno y en } \\
\text { ausencia de éste, } \\
\text { el tutor. }\end{array}$ & $\begin{array}{l}\text { Acuerdo Tutor- } \\
\text { alumno y en } \\
\text { ausencia de } \\
\text { éste, el tutor. }\end{array}$ \\
\hline $\begin{array}{l}\text { Requisitos y plazos } \\
\text { para el Depósito }\end{array}$ & $\begin{array}{l}\text { La Facultad, en } \\
\text { su normativa } \\
\text { interna. }\end{array}$ & $\begin{array}{l}\text { La Comisión } \\
\text { Interna del } \\
\text { Departamento. }\end{array}$ & $\begin{array}{l}\text { La Dirección del } \\
\text { Departamento, } \\
\text { delegando en La } \\
\text { Comisión Interna. } \\
\text { Plazos: el centro }\end{array}$ & $\begin{array}{l}\text { La Dirección del } \\
\text { Departamento, } \\
\text { delegando en La } \\
\text { Comisión Interna. } \\
\text { Plazos: el centro }\end{array}$ \\
\hline $\begin{array}{l}\text { Componentes de } \\
\text { la Comisión } \\
\text { Evaluadora }\end{array}$ & $\begin{array}{l}\text { La Comisión de } \\
\text { TFG del centro, } \\
\text { dentro de los } \\
\text { docentes con } \\
\text { carga en TFG de } \\
\text { las áreas de } \\
\text { conocimiento } \\
\text { compatibles con } \\
\text { el TFG (puede } \\
\text { implicar a más } \\
\text { de un } \\
\text { departamento) }\end{array}$ & $\begin{array}{l}\text { La Comisión } \\
\text { Interna del } \\
\text { Departamento. }\end{array}$ & $\begin{array}{l}\text { La Comisión de } \\
\text { TFG del centro, } \\
\text { sobre propuestas } \\
\text { que emiten los } \\
\text { departamentos. }\end{array}$ & $\begin{array}{l}\text { El Consejo de } \\
\text { Departamento, a } \\
\text { propuesta del } \\
\text { tutor entre los } \\
\text { profesores } \\
\text { adscritos al } \\
\text { grupo TFG. }\end{array}$ \\
\hline $\begin{array}{l}\text { Regulación del } \\
\text { Acto de } \\
\text { presentación. }\end{array}$ & La Facultad & $\begin{array}{l}\text { La Comisión } \\
\text { Interna del } \\
\text { Departamento. }\end{array}$ & $\begin{array}{l}\text { La Dirección del } \\
\text { Departamento, } \\
\text { delegando en La } \\
\text { Comisión Interna. }\end{array}$ & $\begin{array}{l}\text { La Dirección del } \\
\text { Departamento, } \\
\text { delegando en La } \\
\text { Comisión Interna. }\end{array}$ \\
\hline $\begin{array}{l}\text { Criterios de } \\
\text { evaluación }\end{array}$ & La Facultad & $\begin{array}{l}\text { La Comisión } \\
\text { Interna del } \\
\text { Departamento. }\end{array}$ & $\begin{array}{l}\text { La Dirección del } \\
\text { Departamento, } \\
\text { delegando en La } \\
\text { Comisión Interna. }\end{array}$ & $\begin{array}{l}\text { La Dirección del } \\
\text { Departamento, } \\
\text { delegando en La } \\
\text { Comisión Interna. }\end{array}$ \\
\hline
\end{tabular}


De los cuatro centros, en tres se hace delegación de funciones a los departamentos y son las propias comisiones departamentales las que definen todos los aspectos relacionados con el TFG. Esta delegación no está contemplada por la normativa de la US.

Respecto a la asignación del número de trabajos a los departamentos, el criterio es objetivo por cuanto todos los departamentos con docencia en el centro tienen asignados TFG en función de su peso relativo en la titulación. Sin embargo, este criterio puede provocar problemas con aquellos departamentos con escasa carga docente en la titulación (asociado principalmente a asignaturas instrumentales o de formación general) que proponen temas muy específicos de dicha materia por lo que se alejan del objetivo final del TFG. Por ejemplo, un alumno que cursa el Grado de Administración y Dirección de Empresas puede tener la obligación de realizar un trabajo de Derecho Administrativo.

Sobre la asignación del tutor, los alumnos son generalmente asignados, al departamento que desean realizar sU TFG, atendiendo a su expediente académico; en el caso de la Facultad de Turismo y Finanzas y la Facultad de Derecho se introduce la posibilidad de que el alumno llegue a un acuerdo con el profesor para ser tutorizado.

La asignación del tema, se suele imponer el acuerdo profesor-alumno.

En relación a los requisitos y plazos para el depósito, la Facultad de Turismo y Finanzas tiene establecido plazos para el depósito de los TFG para cada una de las convocatorias oficiales. En la Facultad de Ciencias Económicas son las comisiones de TFG de los departamentos quienes establecen las condiciones y en Derecho y Ciencias del Trabajo los plazos para el depósito los dispone el Decanato y las condiciones de entrega, los departamentos. Respecto de los requisitos para el depósito, todos los centros exigen la entrega por parte del estudiante del trabajo, adjuntando en el mismo un anexo con el visto bueno del tutor.

Por último, respecto a las Comisiones Evaluadoras, salvo la Facultad de Turismo y Finanzas, los centros delegan en los departamentos la designación de miembros de las comisiones evaluadoras. De esta manera los trabajos son evaluados por profesores del departamento. En el caso de la Facultad de Turismo y Finanzas, una comisión interna del centro determina el número de comisiones, los profesores que la integran y los trabajos que han de evaluar; la diferencia respecto del resto de centros es que las comisiones son nombradas por áreas de conocimiento y no por departamentos lo que puede repercutir en que comisiones evaluadoras tengan que valorar trabajos sobre temas en los que no son competentes.

\section{METODOLOGÍA}

El trabajo se ha centrado en el área de conocimiento en Ciencias Sociales y Jurídicas de la Universidad de Sevilla, en concreto se han seleccionado la Facultad de Ciencias Económicas y Empresariales (Grado de Administración de Empresas, GADE; Grado de Economía y Grado de Marketing) Facultad de Turismo y Finanzas (Grado de Finanzas y Contabilidad, GFICO) y Grado de Turismo, GT), Facultad de Derecho (Grado de Derecho, GD)) y Facultad de Ciencias del Trabajo (Grado de Relaciones Laborales, GRRLL); los motivos que justifican esta elección son:

- Ser los centros más representativos del área, por su peso en el número de grados, alumnos y profesorado. 
- El hecho de compartir materias en sus diseños curriculares.

- El importante número de dobles grados que implican a dos centros (actualmente se ofertan 9 dobles grados entre estos centro) lo que implica una gran transversalidad

- La existencia de un importante número de profesores que imparten docencia en más de uno de estos centros, por lo que están implicados en la materia de TFG en más de un centro.

Los temas elegidos para debatir con los profesores de los centros elegidos se han obtenido mediante reuniones personales y en grupo, diferenciando edad, categorías profesionales y áreas de conocimiento y a continuación se elabora el listado provisional abierto.

Se obtuvo el siguiente listado de temas que preocupaban a los profesores en el actual desarrollo de la asignatura de TFG:

- La necesidad de una regulación unificada.

- Créditos que pueden asumir los departamentos en cada curso académico por los TFG.

- Obligatoriedad del TFG en los grados, para los profesores y los alumnos.

- Dedicación y reconocimiento de créditos de los TFG tutorizados.

- Asignación de los tutores a los alumnos.

- Método de trabajo seguido por el profesor.

- Evaluación.

Se realizan un total de cinco reuniones, una en grupo de 40 profesores de los diferentes centros implicados y cuatro en grupo de 6 profesores, de las comisiones de seguimiento de los TFG de los distintos centros.

Las reuniones se desarrollan con el listado de temas elaborado pero abiertas a cualquier otro tema de interés en el momento del debate. Las opiniones vertidas fueron recogidas por los autores del trabajo y son las que presentamos a continuación.

\section{EL TRABAJO FIN DE GRADO A DEBATE}

Del apartado referente a la normativa se destaca que la normativa nacional (Real Decreto 1393/2007) no entra en exponer los aspectos de desarrollo de lo que tiene que ser un TFG y lo delega a las normativas internas de cada universidad. En el caso de la Universidad de Sevilla, como ha quedado expuesto, disponemos de una normativa general y de una normativa por centro.

De las diferentes reuniones que se han mantenido con el colectivo de profesores y comisiones de seguimiento, exponemos las opiniones vertidas en el debate respecto a:

\section{A. La necesidad de una regulación unificada}

Tomando como referencia al Departamento de Contabilidad y Economía Financiera de la Universidad de Sevilla, actualmente imparte docencia, de manera significativa, en los cuatro centros implicados en el trabajo por lo que debe gestionar cuatro normativas con elementos diferenciados importantes que afectan, no tanto al desarrollo y realización del TFG, sino a los aspectos de gestión, admisión y 
procedimiento de asignación de los alumnos a los departamentos y a los tutores (ver tabla 1).

\section{Tema a debate: ¿Normativa única o diversidad normativa por centros?}

Existe unanimidad de criterio, entre los profesores, respecto a que la normativa de la universidad debería unificar al menos aquellos componentes comunes a todos los centros, fundamentalmente las directrices de gestión de admisión, así como el procedimiento de asignación de los alumnos a los departamentos. Esta unificación podría ser bajo un enfoque de universidad o bien de áreas de conocimiento.

Derivado de las diferencias normativas por centros que existen en la actualidad, los departamentos, hasta que no termina el proceso de asignación, desconocen cuál es el número total de alumnos que van a asumir en el curso académico y los profesores desconocen el número definitivo de alumnos que van tutorizar.

No obstante, también están de acuerdo que una normativa unificada para toda la universidad podría generar disfunciones en la gestión y rigideces en la ejecución del TFG; la singularidad y los elementos diferenciadores de cada una de las áreas de conocimiento son indudables; las áreas técnicas y las experimentales difieren de manera clara del área de ciencias sociales y jurídicas y de humanidades. Además en las áreas técnicas, como ya quedo expuesto, este tipo de trabajos no representan una novedad ya que desde la década de los 50 se viene realizando el denominado Proyecto Fin de Carrera; equivalente al TFG actual.

Existe un reconocimiento al papel que actualmente están desempeñando los diferentes órganos de gestión (universidad, centros y departamentos) en la difusión de la normativa de los TFG, pero de manera explícita los centros que son los que están jugado el papel más relevante.

\section{B. Créditos que pueden asumir los departamentos en cada curso académico por los TFG}

Teniendo en cuenta la situación actual que están viviendo los departamentos, donde en los últimos años no sólo no se ha contratado nuevo profesorado, sino que por el contrario se está reduciendo vía no renovación de contratos, abandono de profesores asociados, jubilaciones, finalización de contratos de becarios... y además, se está incrementando la carga en el Plan de Organización Docente con materias nuevas procedentes de los nuevos grados y de los master oficiales; si a ello se le añade que no existe una limitación clara en la adscripción de los alumnos a los departamentos para la realización de los TFG (ver tabla 2), el resultado es que va a ser difícil cumplir con la Norma de Modulación de Dedicación Académica del Profesorado de la Universidad de Sevilla (artículo 8; Acuerdo 8.6 CG 30/4/13), que no entrando en confrontación directa con la Nota Sobre el Régimen de Dedicación del Profesorado Universitario del Ministerio de Educación (02.07.12) que fijaba la dedicación entre 16 y 32 créditos; si acuerda que (art. 8.2):

2. Para aminorar la dedicación del profesorado cuyo máximo es de 320 horas anuales de docencia presencial, ello sin menoscabo de la prestación del servicio y respetando lo dispuesto en los artículos anteriores, en las áreas de conocimiento de los departamentos se aplicará, en función del encargo docente, la medida de modulación que pueda corresponder de entre las siguientes: 
a) El máximo de dedicación académica de todo el profesorado serán 240 horas anvales en aquellas áreas de conocimiento en las que con esta limitación puede cubrirse todo el encargo docente.

b) El máximo de dedicación académica de todo el profesorado con título de doctor serán 240 horas anuales en aquellas áreas de conocimiento en las que con esta limitación puede cubrirse todo el encargo docente.

\begin{tabular}{|l|l|l|}
\hline \multicolumn{3}{|c|}{ Tabla 2. Número de TFG matriculados y asignación a departamentos } \\
\hline Centro & $\begin{array}{l}\text { Alumnos } \\
\text { Matriculados }\end{array}$ & Asignación a los departamentos \\
\hline FCEYE & Indefinido & $\begin{array}{l}\text { Indefinido, en función de carga docente relativa (número } \\
\text { de créditos y número de grupos de las asignaturas en los } \\
\text { títulos). }\end{array}$ \\
\hline FTF & Indefinido & $\begin{array}{l}\text { Indefinido, en función de carga docente relativa (número } \\
\text { de créditos y número de grupos la las asignaturas en los } \\
\text { títulos). }\end{array}$ \\
\hline FD & Indefinido & En función de carga docente \\
\hline FCT & Indefinido & En función de carga docente \\
\hline
\end{tabular}

Si tomamos como referencia la Facultad de CEYE y la de TF, la evolución del número de TFG tutorizados desde el curso 2012-13 al 2015-16 (tabla 3), se observa que han experimentado un crecimiento muy significativo, pasando de 514 en el curso 2012-13 a 1716 en el curso 2015-16; destacando el Grado de Finanzas y Contabilidad (GFICO) de 111 a 342 y el Grado de Administración de Empresas (GADE) de 210 a 840.

\begin{tabular}{|c|c|c|c|c|c|c|c|c|c|c|c|c|}
\hline \multicolumn{13}{|c|}{ Tabla 3. TFG asignados por Grados } \\
\hline & $\begin{array}{c}2012 / \\
13\end{array}$ & $\% \mathrm{~V}$ & $\begin{array}{c}2013 / \\
14\end{array}$ & $\% \mathrm{~V}$ & $\% \mathrm{H}$ & \begin{tabular}{|c|}
$2014 /$ \\
15
\end{tabular} & $\% \mathrm{~V}$ & $\% \mathrm{H}$ & $\begin{array}{c}2015 / \\
16\end{array}$ & $\% \mathrm{~V}$ & $\% \mathrm{H}$ & $\begin{array}{c}\Delta \\
2012 / 16\end{array}$ \\
\hline $\mathrm{FICO}$ & 111 & $22 \%$ & 271 & $25 \%$ & $144 \%$ & 351 & $24 \%$ & $30 \%$ & 342 & $20 \%$ & $-3 \%$ & $208 \%$ \\
\hline TURISMO & 161 & $31 \%$ & 213 & $19 \%$ & $32 \%$ & 269 & $19 \%$ & $26 \%$ & 335 & $20 \%$ & $25 \%$ & $108 \%$ \\
\hline ADE & 99 & $19 \%$ & 266 & $24 \%$ & $169 \%$ & 350 & $24 \%$ & $32 \%$ & 498 & $29 \%$ & $42 \%$ & $403 \%$ \\
\hline ECONOMÍA & 25 & $5 \%$ & 88 & $8 \%$ & $252 \%$ & 113 & $8 \%$ & $28 \%$ & 128 & $7 \%$ & $13 \%$ & $412 \%$ \\
\hline MARKETING & 12 & $2 \%$ & 31 & $3 \%$ & $158 \%$ & 62 & $4 \%$ & $100 \%$ & 83 & $5 \%$ & $34 \%$ & $592 \%$ \\
\hline $\begin{array}{l}\text { RELACIONES } \\
\text { LABORALES }\end{array}$ & 106 & $21 \%$ & 212 & $2 \%$ & $100 \%$ & 248 & $3 \%$ & $17 \%$ & 275 & $3 \%$ & $11 \%$ & $159 \%$ \\
\hline $\mathrm{ADE}+\mathrm{DERECHO}$ & $\mathrm{ND}$ & & 17 & $2 \%$ & & 46 & $3 \%$ & $171 \%$ & 55 & $3 \%$ & $20 \%$ & $224 \%$ \\
\hline Total & 514 & $100 \%$ & 1.098 & $100 \%$ & $114 \%$ & 1.439 & $100 \%$ & $31 \%$ & 1716 & $100 \%$ & $19 \%$ & $234 \%$ \\
\hline
\end{tabular}

En el caso de los departamentos, si tomamos como referencia el Departamento de Contabilidad y Economía Financiera ha supuesto pasar de 59 trabajos en el curso 2012-13 a 239 en curso 2015-16 (tabla 4). 


\begin{tabular}{|l|c|c|c|c|c|c|c|c|c|c|c|c|}
\hline \multicolumn{7}{|c|}{ Tabla 4. TFG asignados al Departamento de Contabilidad de la Universidad de Sevilla } \\
\hline & $\begin{array}{c}\mathbf{2 0 1 2} \\
\mathbf{1 1 3}\end{array}$ & $\mathbf{\% V}$ & $\begin{array}{c}\mathbf{2 0 1 3} \\
/ \mathbf{1 4}\end{array}$ & $\mathbf{\% V}$ & $\mathbf{\% H}$ & $\begin{array}{c}\mathbf{2 0 1 4} \\
/ \mathbf{1 5}\end{array}$ & $\mathbf{\% V}$ & $\mathbf{\% H}$ & $\begin{array}{c}\mathbf{2 0 1 5} \\
/ \mathbf{1 6}\end{array}$ & $\mathbf{\% V}$ & $\mathbf{\% H}$ & $\begin{array}{c}\mathbf{2 0 1 2} \\
/ \mathbf{1 6}\end{array}$ \\
\hline FICO & 29 & $49 \%$ & 76 & $51 \%$ & $162 \%$ & 93 & $\mathbf{4 7 \%}$ & $22 \%$ & 86 & $36 \%$ & $-8 \%$ & $197 \%$ \\
\hline TURISMO & 10 & $17 \%$ & 15 & $10 \%$ & $50 \%$ & 17 & $9 \%$ & $13 \%$ & 26 & $11 \%$ & $53 \%$ & $160 \%$ \\
\hline ADE & 15 & $25 \%$ & 42 & $28 \%$ & $180 \%$ & 60 & $30 \%$ & $43 \%$ & 94 & $39 \%$ & $57 \%$ & $527 \%$ \\
\hline ECONOMÍA & 1 & $2 \%$ & 2 & $1 \%$ & $100 \%$ & 7 & $4 \%$ & $250 \%$ & 5 & $2 \%$ & $-29 \%$ & $400 \%$ \\
\hline MARKETING & 0 & $0 \%$ & 1 & $1 \%$ & $100 \%$ & 3 & $2 \%$ & $200 \%$ & 7 & $3 \%$ & $133 \%$ & $700 \%$ \\
\hline $\begin{array}{l}\text { RELACIONES } \\
\text { LABORALES }\end{array}$ & 4 & $7 \%$ & 10 & $7 \%$ & $150 \%$ & 13 & $7 \%$ & $30 \%$ & 14 & $6 \%$ & $8 \%$ & $250 \%$ \\
\hline ADE+DERECHO & 0 & $0 \%$ & 2 & $1 \%$ & $200 \%$ & 5 & $3 \%$ & $150 \%$ & 7 & $3 \%$ & $40 \%$ & $700 \%$ \\
\hline Total & $\mathbf{5 9}$ & $100 \%$ & $\mathbf{1 4 8}$ & $100 \%$ & $151 \%$ & $\mathbf{1 9 8}$ & $\begin{array}{c}100 \\
\%\end{array}$ & $34 \%$ & $\mathbf{2 3 9}$ & $100 \%$ & $21 \%$ & $305 \%$ \\
\hline
\end{tabular}

Tema a debate: ¿Cuál es el límite de carga en créditos que pueden asumir los departamentos por los TFG en cada curso académico?

Los profesores defienden la existencia de un límite máximo de carga en créditos a asumir por cada departamento y número de TFG asumidos por profesor; por el contrario no comparte la limitación de un máximo de alumnos matriculados en la asignatura de TFG por centro y curso académico.

La propuesta pasa por reconocer que el criterio a tener en cuenta para fijar el número máximo de alumnos que pueden matricularse en la asignatura TFG y en su asignación a los departamentos debería ser la diferencia entre la carga total reconocida en el Neoplan de la Universidad y la asumida realmente y no aplicar exclusivamente los criterios de elección voluntaria de los alumnos o/y carga docente relativa (número de créditos y número de grupos de las asignaturas en los títulos).

Un obstáculo añadido es que al no existir un criterio temporal homogéneo de asignación de los TFG a los departamentos, estos llegan de manera atemporal y asimétrica; lo que provoca que profesores tengan que asumir necesariamente más carga académica durante el curso académico.

\section{Obligatoriedad del TFG en los grados, para los profesores y los alumnos}

Entre los cambios que se han producido en la universidad española en esta última década destaca los esfuerzos realizados, por parte del profesorado, por la implantación de metodologías de participación activa (aprendizaje basado en problemas, aprendizaje basado el proyectos...) lo que ha repercutido en el desarrollado de competencias y habilidades de los alumnos relacionadas con la realización de trabajos y su defensa.

Asimismo, en algunos grados se han incorporado en su diseño curricular materias que por contenido y metodologías desarrollan idénticas competencias que la materia de TFG; Así en el Plan de Estudios de ADE y FICO encontramos dos asignaturas con contenidos que podrían perfectamente estar solapándose con el TFG; en concreto, estas asignaturas son Creación de Empresas ( $4^{\circ}$ curso, 6 créditos, obligatoria) y Plan de Empresa ( $4^{\circ}$ curso, 12 créditos, optativa). Dos materias, que en la Universidad de Sevilla 
tienen recogido dentro de sus programas el desarrollo de la competencia de realización de un trabajo y su defensa.

La asignatura de Creación de Empresas contempla el desarrollo de las siguientes competencias específicas:

- Habilidades de redacción de proyectos de relativa complejidad.

- Habilidades de búsqueda de información específica y especializada.

- Capacidad de defensa de proyecto.

- Capacidad de trabajo en grupo con propósito determinado y plazo limitado.

- Capacidad de comprender la realidad empresarial de forma integral, coordinada y coherente.

La asignatura de Plan de Empresa tiene entre sus objetivos docentes específicos: Hacer comprender al alumno como se elabora un Plan de Empresa y la importancia del mismo en el ámbito emprendedor y de la empresa innovadora y en particular:

- Buscar e identificar ideas de negocio.

- Búsqueda de información sobre la idea de negocio.

- Conocimiento del entorno de la idea de negocio.

- Evaluar la viabilidad de la idea de negocio como producto para un entorno concreto.

- Búsqueda de información para la elaboración del Plan de Empresa.

- Elaboración del Plan de Empresa.

La inclusión de la realización de un trabajo, en temáticas que son compatibles con los temas asignados en los TFG, hacen que el contenido de las dos asignaturas mencionadas puedan cubrir la formación del alumno en las competencias que se asignan al TFG; sobre todo por su enfoque multidisciplinar.

Tema a debate: ¿̇Es realmente necesaria la obligatoriedad de la asignatura TFG?

La opinión está dividida; por una parte se participa de la idea de que siga siendo obligatoria pero con una mejora considerable en el reconocimiento de los créditos para el tutor. Otro importante número de opiniones van en la dirección de que si los resultados de los trabajos siguen teniendo tan baja calidad, como los presentados hasta el presente curso y, siendo mayoritaria la opinión de que no han cumplido con los objetivos planteados, sería mejor que esta asignatura no fuese obligatoria y que se incorporara dentro de la categoría de asignaturas optativas o que se extinguiera.

\section{Dedicación y reconocimiento de créditos de los TFG tutorizados}

Cómo se enfrenta la Universidad de Sevilla al difícil equilibrio que significan las restricciones presupuestarias, la no contratación de nuevo profesorado, el incremento de carga docente y paralelamente la modulación de la dedicación académica del profesorado; la respuesta ha sido no reconociendo o haciéndolo de manera muy limitada la dedicación del profesorado a ciertas actividades docentes, de gestión e investigación. Este no reconocimiento está generando una actitud crítica frente a la institución que a nivel departamental se traduce en una desmotivación, abandono o no colaboración del profesorado en las tareas de gestión y, además, amenaza con 
romper el frágil equilibrio solidario entre los gestores de los departamentos y el profesorado. Paralelamente se está generando un enroque hacia el individualismo del profesor que sólo valora aquello que está reconocido en la lista de méritos para la promoción universitaria.

El debate se centra en la estabilidad, uniformidad normativa en el reconocimiento de créditos entre los centros y titulaciones y alumnos-profesores; y además que se reconozca un número de créditos más real por TFG tutorizado de lo que hasta ahora se viene considerando.

Tema a debate: ¿Por qué no existe estabilidad normativa en el reconocimiento de créditos?

En la Universidad de Sevilla hemos tenido dos tipos de reconocimiento; comenzamos en el curso académico 2012-13 reconociendo los créditos de tutorización, asignados en el Plan de Asignación del Profesorado (PAP), en el mismo curso que se realizaba el TFG, lo que permitía fijar, de manera consensuada, la dedicación individualizada de cada profesor sin sobrepasar la modulación de dedicación docente. El método planteaba el inconveniente que profesores a los que se les reconocía la carga por TFG no llegaban a desarrollarla por falta de alumnos 0 , por el contrario, si existía más carga de la reconocida se procedía a su reparto con criterios departamentales; como asignarlo a aquellos profesores con menor carga total en SU PAP, lo que causaba malestar en el profesorado afectado debido a que era una carga sobrevenida.

Para el curso académico 2013-14 la Universidad de Sevilla, debido a la obligación de dar cumplimiento a su Norma de Modulación de Dedicación Académica del Profesorado, se vio en la necesidad de diferir el reconocimiento de la carga de los TFG para el curso 2014-15; el motivo estaba fundamentado en la convivencia de los dos Planes de Estudios vigentes (licenciaturas y grados) que para el curso 2013-14 producía un incremento de créditos en la dedicación docente total de la universidad de 15000 créditos y para el curso 2014-15, al desaparecer el efecto licenciatura, provocaba una reducción equivalente. Este diferimiento ha generado, en muchos departamentos de la Universidad para el curso 2013-14, un falso positivo en holguras. En el caso del Departamento de Contabilidad, que para el curso 2013-14 no estábamos afectados por el efecto licenciatura, pasamos de tener una carga media por profesor en el curso 2012-13 de 22 créditos a 19 créditos en el curso 2013-14; para a continuación (cursos 2014-15 y 2015-16) igualar o sobrepasar los 24 créditos. Por lo tanto, en cada curso académico los profesores están tutorizando los TFG que se computarán en el siguiente curso, lo que permite a los departamentos conocer de forma real la carga que se le va a asignar a cada profesor. De otra parte, al no estar definido claramente, en cada curso, el límite de adscripción de los alumnos y no poderlo controlar ya que viene impuesto por los centros, los profesores se van a encontrar que muchos de ellos van a sobrepasar su límite de modulación (24 créditos) y, además, paralelamente tendrán que asumir más tutorizaciones ya que en los últimos cursos académicos el número de matriculados en TFG se está incrementado de forma considerable (ver tabla 3). Por todo ello, los profesores solicitan estabilidad normativa y, como se apuntó, fijar límites de créditos de TFG asumidos por los departamentos.

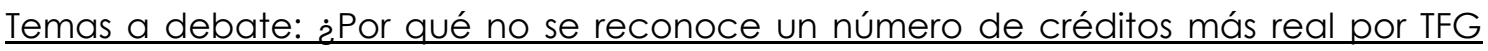
tutorizado? ¿Por qué el número de créditos reconocidos al alumno por la materia de IFG no coincide con el reconocimiento de la carga en PAP para el profesorado?

Uno de los aspectos más controvertidos en el debate de los TFG hace referencia a la relación entre el tiempo en horas de dedicación que están asumiendo los profesores 
en cada TFG y el reconocimiento real en créditos. La opinión del profesorado es que la labor de tutela que realizan no está siendo reconocida adecuadamente.

El reconocimiento de la tutorización de la asignatura de TFG (curso académico 201213) en la Universidad de Sevilla se realiza mediante Resolución Rectoral de 8 de marzo de 2012 en la que se reconocía una carga fija dependiente del número de grupos aprobados que se ha de repartir entre departamentos/profesores, independientemente del número de alumnos matriculados. En términos generales se tradujo en un reconocimiento de $0^{\prime} 2$ créditos por cada TFG tutorizado. Este reconocimiento implicaba que el tiempo que debería dedicar un profesor a tutorizar un TFG era de dos horas; lo que produjo un estado de desconcierto y, parte del profesorado, valoró la importancia de su tarea en función del valor que le concedió la institución; su respuesta fue aplicar el tiempo de forma estricta; lo que se tradujo en un malestar en la relación con el alumno y un sentimiento, por parte del alumno, de engaño y abandono.

Para el curso 2013/2014 la Universidad de Sevilla en su Normativa Transitoria sobre Dedicación Académica del Profesorado reconoció una carga fija de 5 horas 10,5 créditos) por cada 6 ECTS en TFG. Este cambio no ha mejorado el ambiente existente, ya que ha sido más cualitativo que cuantitativo.

Recogiendo las opiniones del profesorado sobre su dedicación en la tutorización de TFG, durante los últimos cursos académicos, el perfil tipo por curso académico sería: cada profesor está tutorizando una media 4 TFG y el número de horas totales dedicadas a cada TFG está entre 10 y 20 horas, computando no sólo las horas de tutoría presencial sino también las revisiones, correcciones y preparación para la defensa.

En el debate se destaca el hecho de que al alumno se le está reconociendo por la asignatura de TFG un total de 6 créditos y no existe uniformidad en su reparto de los créditos reconocidos entre presencialidad y no en las diferentes titulaciones y la presencialidad no coincide con el reconocimiento al profesor (tabla 5).

\begin{tabular}{|l|l|c|}
\hline \multicolumn{2}{|c|}{ Tabla 5. Créditos reconocidos por titulación } \\
\hline Titulación & Presencial/No presencial & Créditos reconocidos \\
\hline Grado ADE & Presencial & 1,8 \\
\cline { 2 - 3 } & No presencial & 3,6 \\
\cline { 2 - 3 } & Otros & $0^{\prime} 6$ \\
\hline \multirow{4}{*}{ Grado Derecho } & Presencial & 0,6 \\
\cline { 2 - 3 } & No presencial & 5,4 \\
\cline { 2 - 3 } & Otros & 0 \\
\hline \multirow{3}{*}{ Grado } & Presencial & 1,8 \\
\cline { 2 - 3 } & No presencial & 3,6 \\
\cline { 2 - 3 } & Otros & 0,6 \\
\hline \multirow{3}{*}{ Erado } & Presencial & 1,8 \\
\cline { 2 - 3 } & No presencial & 3,6 \\
\cline { 2 - 3 } & Otros & 0,6 \\
\hline \multirow{3}{*}{ Grado FICO } & Presencial & 0,2 \\
\cline { 2 - 3 } & No presencial & 3,6 \\
\cline { 2 - 3 } & Otros & 2,2 \\
\hline \multirow{3}{*}{ Grado Turismo } & Presencial & 0,2 \\
\cline { 2 - 3 } & No presencial & 3,6 \\
\cline { 2 - 3 } & Otros & 2,2 \\
\hline \multirow{3}{*}{ Grado RRLL } & Presencial & 1,8 \\
\cline { 2 - 3 } & No presencial & 3,6 \\
\cline { 2 - 3 } & Otros & 0,6 \\
\hline
\end{tabular}


En definitiva, el profesorado está demandando mayor uniformidad, a nivel de centros, en la actual normativa sobre los TFG y reconocimiento real de créditos a los profesores tutores.

\section{E. Método de trabajo seguido por el profesor}

Cuando los profesores se enfrentan por primera vez a la tutorización de un TFG, la duda que manifiestan es que no ha quedado claro lo que es un TFG; las normativas no son de gran ayuda. La normativa nacional (Real Decreto 1393/2007 de 29 de octubre) en su art. 12.7 apunta que deberá realizarse en la fase final del plan de estudios y estar orientado a la evaluación de competencias asociadas al título. Una definición suficientemente general y borrosa para que cada universidad, cada centro o facultad y plan de estudios de TFG pueda desarrollar su propia definición y marco de acción (Bartolomé et al., 2012).

La normativa de la Universidad de Sevilla (Acuerdo 5.3/CG 21-12-09), que ha sido reproducida por las normativas internas de los centros, no es mucho más explícita: El Trabajo Fin de Carrera consistirá en la realización por parte del estudiante, bajo la dirección de un tutor, de un proyecto, memoria o estudio sobre un tema de trabajo que se le asignará y en el que desarrollará y aplicará conocimientos, capacidades y competencias adquiridos en la titulación (art.1).

Los temas que han suscitado debate a esta definición hacen referencia a:

Tema a debate: $\dot{2}$ El término Tutorización equivale a lo que las propias normativas (universidad y centros) definen como la función de Dirección o se acerca más al concepto de Mentorización?

La normativa de la Universidad de Sevilla apunta en su art.1, que el TFG lo realizará el estudiante bajo la Dirección de un tutor y en el art.5 determina que la función del tutor consistirá en orientar al estudiante durante la realización del trabajo, supervisarlo y velar por el cumplimiento de los objetivos fijados. Si esta concepción la vinculamos al término técnico de lo que implica Dirección, su contenido no coincide con lo expuesto por la normativa, ya que Dirigir implica guiar y llevar la actividad encomendada a un fin, dar reglas, aconsejar u orientar; lo que conlleva: toma de decisiones, definición de problemas, análisis de problemas, evolución de diferentes alternativas, elección entre alternativas, aplicar decisiones, integración, motivación, comunicación y asunción de responsabilidad.

Los profesores opinan que es imposible realizar una actividad pura de dirección con el tiempo reconocido $(0,5$ créditos) y se inclinan por adscribirla dentro de lo que se denomina mentorización. Esta contempla la función del profesor como consejero o guía para el estudiante; es más centrada en la transferencia por la experiencia y los conocimientos del mentor, siendo la responsabilidad del resultado exclusivamente del alumno. Una característica importante del mentor es que tiene que ser una persona de experiencia y ser aceptada y reconocida por el alumno.

Tema a debate: ¿Estamos ante un TFG orientado a la formación investigadora, a la continuidad de la formación académica o a la preparación profesional?

La normativa de la Universidad de Sevilla (art.1) deja abierta la posibilidad a cualquiera de las modalidades ya que apunta que podrá ser un proyecto, memoria o 
estudio sobre un tema; en el que el alumno desarrollará y aplicará conocimientos, capacidades y competencias adquiridas en la titulación.

Los profesores dudan que los alumnos durante el Grado hayan adquirido competencias vinculadas a la investigación; por lo tanto, asignan el TFG al aprendizaje del alumno y evaluación de competencias y no centrado en la obtención de resultados de investigación. La revisión de las memorias de verificación de los diferentes grados; se observa que entre las competencias enumeradas no se encuentran referencias directas al desarrollo específico de competencias de investigación. Los profesores están orientando el TFG a un mixto de formación académica-profesional-investigación y apuestan por un enfoque de preparación profesional.

\section{Tema a debate: ¿ TFG Monodisciplinar o Multidisciplinar?}

Como se ha apuntado en el párrafo anterior, la norma, en su art.1, dicta que en el TFG se desarrollará y aplicará conocimientos, capacidades y competencias adquiridas en la titulación; y el objetivo, que se recoge en el programa de la asignatura, es que el TFG debe formar al alumno en el desarrollo de su capacidad de análisis en la transversalidad de las materias del grado. Por lo tanto el TFG debería integrar conocimientos adquiridos a lo largo de las diferentes materias que el alumno ha cursado por lo que el TFG debería ser multidisciplinar. Como apuntan Bartolomé et al. (2012), el TFG multidisciplinar debe ser uno de los principales retos y dificultades, tanto para los alumnos, que generalmente están acostumbrados a trabajar con conocimientos parcelados; así como para el profesor que suele estar especializado en líneas de trabajo muy concretas.

Los profesores, aunque reconocen que los TFG deberían tener un contenido multidisciplinar, la realidad actual es que priman Ios TFG monodisciplinares; ya que, aunque dan la opción a varios temas, suele proponer temas que están en la base de su propia especialización. Los profesores lo justifican atendiendo al bajo reconocimiento en créditos de la actividad y además, teniendo en cuenta que apuestan por el papel de mentor, es más lógico que el profesor se apoye en aquellos temas que domina y que le permiten la maximización del tiempo de supervisión.

\section{F. Evaluación}

La normativa nacional da la posibilidad de utilizar procesos de evaluación alternativos a la exposición oral en sesión pública. Recordamos que el art. 12.3 del RD. 1393/2007, referente a los títulos de grado, indica que estas enseñanzas concluirán con la elaboración y defensa de un trabajo de fin de grado. La Universidad de Sevilla le ha dado carácter de defensa pública con tribunal constituido por tres titulares y tres suplentes con plena capacidad de evaluación y son los centros los que deciden la obligatoriedad o no del informe del tutor, pero siempre como elemento de referencia.

Los profesores consideran que el proceso de evaluación mediante tribunales se ha traducido en una sobrecarga de dedicación no reconocida, ya que obliga, adicionalmente a la tutorización, a la lectura y evaluación de un número no definido de TFG a evaluar en tribunales.

Tema a debate: ¿Las comisiones evaluadoras debe estar formadas exclusivamente por profesores conocedores de la materia objeto de evaluación? 
La normativa no indica nada al respecto, por lo que cualquier profesor que imparta docencia en el grado, al que pertenece el trabajo, está habilitado para poder evaluarlo. Por lo tanto queda a la discreción de los centros su formación; en el caso de la Facultad de Turismo y Finanzas, una comisión interna determina el número de comisiones evaluadoras, los profesores que la integran y los trabajos que han de evaluar. La diferencia respecto del resto de centros es que las comisiones son nombradas por áreas de conocimiento y no por departamentos lo que puede repercutir, como así ha ocurrido, que comisiones evaluadoras entren a calificar trabajos sobre temas en los que no son competentes.

La opinión recogida se inclina en la dirección de que la comisión debería estar formada por profesores conocedores de la materia, tanto si el TFG es monodisciplinar como si es multidisciplinar.

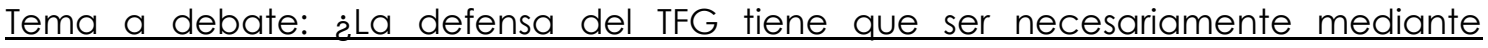
exposición oral en sesión pública?

Como ya se ha apuntado, la normativa de la Universidad de Sevilla y la de los centros así lo indican. Pero además, los miembros del tribunal (tres titulares y tres suplentes) deben tener plena capacidad docente y que impartan o hayan impartido docencia en el grado.

La opinión de los profesores es que la pertenencia a estas comisiones no está reconocido en ningún apartado de la normativa, teniendo en cuenta que hay que leer y evaluar cada trabajo que se defiende en la comisión y proponen que se flexibilice la modalidad de evaluación pudiendo incorporar cambios como:

- Que la evaluación real la realice el tutor; el cual en vez de dar sólo el visto bueno al trabajo, realice un informe de evaluación que sea vinculante para la comisión; el papel de la comisión sería evaluar las habilidades de la defensa del trabajo.

- Otra opción sería, la defensa del trabajo mediante exposición en panel; el papel de la comisión sería valorar la realización del panel y la realización de preguntas al alumno frente al panel.

\section{CONCLUSIONES}

La asignatura de TFG, desde su incorporación a los nuevos Planes de Estudio, no ha pasado desapercibida para nuestros centros, departamentos y especialmente para los profesores. Actualmente el debate está abierto y abarca tanto los aspectos puramente normativos como la aportación que esta materia está realizando en la formación del alumno.

El colectivo profesores universitarios es consciente de la difícil situación por la que están pasando nuestras universidades, con restricciones presupuestarias y la imposibilidad de contratación y promoción de profesorado; lo que ha implicado incremento de carga docente, modulación de la dedicación académica del profesorado y el no reconocimiento de la dedicación de su profesorado, no sólo a ciertas actividades docentes sino también a muchas actividades de gestión e investigación

LOS TFG han formado parte de ese conjunto de sumandos no reconocidos; lo que ha hecho que el debate en torno a su regulación e implicación en la carga docente de los profesores sea motivo de debate y de propuestas de mejora. 
Como principales conclusiones al debate que se ha propuesto en nuestras centros de referencia resaltar el profundo descontento que actualmente existe en torno a los TFG entre los profesores participantes (profesores-gestores, profesores-tutores y profesoresevaluadores) en parte por la incertidumbre derivada de la normativa reguladora, en parte por la amplia discrecionalidad que tienen tanto los tutores como evaluadores en el momento de asumir su rol dentro del proceso.

Tras recoger las opiniones de los profesores sobre aquellos aspectos que se han señalado como más sensibles al debate; las propuestas realizadas son:

- Los profesores están de acuerdo que una normativa unificada para toda la universidad podría generar disfunciones en la gestión y rigideces en la ejecución del TFG; pero existe unanimidad de criterio respecto a que la normativa de la universidad debería unificar al menos aquellos componentes comunes a todos los centros, fundamentalmente las directrices de gestión de admisión, así como el procedimiento de asignación de los alumnos a los departamentos. Esta unificación podría ser bajo un enfoque de universidad o bien de áreas de conocimiento.

- El criterio a tener en cuenta para fijar el número máximo de alumnos que pueden matricularse en la asignatura TFG y su asignación a los departamentos, debe ser la diferencia entre la total reconocida por la Universidad y la real; y no aplicar exclusivamente los criterios de elección voluntaria de los alumnos o/y carga docente relativa (número de créditos y número de grupos de las asignaturas en los títulos).

- Debe existir estabilidad y uniformidad normativa en el reconocimiento de créditos entre los diferentes centros; además debe reconocerse un número de créditos más real de lo que hasta ahora se viene considerando.

- El papel del tutor se adscribe mejor a la figura de mentor que a la de director ya que es imposible realizar una actividad pura de dirección con los créditos reconocidos.

- El TFG debe estar orientado a la preparación profesional y la inserción laboral.

- El TFG debería integrar conocimientos adquiridos a lo largo de las diferentes materias que ha cursado en el grado; por lo tanto debería ser más multidisciplinar.

- La opinión está dividida sobre la obligatoriedad del TFG; por una parte se participa de la idea de que siga siendo obligatoria pero con una mejora considerable en el reconocimiento de los créditos para el tutor. Pero teniendo en cuenta la baja calidad de los resultados obtenidos se propone la no obligatoriedad e incluso su desaparición.

- Las comisiones evaluadoras deben estar formadas por profesores conocedores de la materia objeto de evaluación.

- La defensa del TFG no tiene por qué ser necesariamente mediante exposición oral en sesión pública.

- Que la evaluación sea compartida, que el tutor evalúe mediante un informe el desarrollo y contenido y la comisión sobre su defensa.

\section{REFERENCIAS}

Acuerdo 5.3/CG 21-12-09. Normativa Reguladora de los Trabajos Fin de Carrera. Universidad de Sevilla. 
Bartolomé, A.; Carmona, M.; Ferrer, V.; Folgueiras, P.; Freixa, M.; García-Bores, Jm.; Nuri, A.; Padrós, M; Ramis, R. y Soria, V. (2012). El Trabajo Fin de Grado. Guía para Estudiantes, docentes y agentes colaboradores. Mc Graw-Hill.

Jessop, M. (2005). Teaching, Learning and Research in Final Year Humanities Computing Student Project" Literary and Linguistic Computing, Vol. 20, No. 3. Oxford University Press Pp. 295-311.

Ley de 20 de julio de Ordenación de las Enseñanzas Técnicas. BOE 20-07-1957.

Mateo, J.; Escofet, A.; Martínez, F.; Ventura, J. y Vlachopoulos, D. (2012): "The Final Year Project (FYP) in social sciences: Establishment of its associated competences and evaluation standards" Studies in Educational Evaluation n 38. Pp. 28-34.

http://www.sciencedirect.com/science/article/pii/S0191491X11000575

Ministerio de Educación, Cultura y Deporte (2004): "Evolución del Sistema Educativo Español". Madrid. http://vom.vib.cat/digitalAssets/202/202199_6.pdf

Real Decreto 1393/2007, de 29 de octubre. Ordenación de las enseñanzas universitarias oficiales. Ministerio de Educación y Ciencia.

Real Decreto 861/2010, de 2 de julio, por el que se modifica el Real Decreto 1393/2007, de 29 de octubre, por el que se establece la ordenación de las enseñanzas universitarias oficiales. Ministerio de Educación y Ciencia.

Rekalde Rodríguez, I. (2011): "¿Cómo afrontar el trabajo fin de grado? Un problema o una oportunidad para culminar con el desarrollo de las competencias". Revista Complutense de Educación Vol. $22 n^{\circ}$ 2. Universidad Complutense de Madrid, Facultad de Filosofía y Ciencias de la Educación Pp 179-193.

Valderrama, E.; Rullán, M.; Sánchez, F.; Pons, J.; Cores, F. y Bisbal, J. (2009): "La evaluación de las competencias en los Trabajos de Fin de Estudios" XV Jornadas de Enseñanza Universitaria en Informática. Barcelona 8-10 de julio http://jenui2009.fib.upc.edu/ 